\title{
Mecanismos de Atenção Selectiva na Perturbação de Hiperactividade com Déficit de Atenção
}

\section{Selective Attention Mechanisms Applied in Attention Deficit Hyperactivity Disorder}

\author{
Joaquim M. F. Ramalho*, ${ }^{*}$, M. Mar García-Señorán ${ }^{b}, \&$ Salvador G. González ${ }^{b}$ \\ ${ }^{a}$ Universidade Fernando Pessoa \& ${ }^{b}$ Universidad de Vigo
}

\begin{abstract}
Resumo
O déficit atencional é o factor primordial da Transtorno do Deficit de Atenção com ou sem Hiperactividade (TDA/H). Neste artigo procura-se comprovar as dificuldades na atenção selectiva dos sujeitos com TDA/ $\mathrm{H}$ em comparação com os sujeitos sem TDA/H, procurando verificar quais os mecanismos deste processo da atenção que estão mais afectados nos sujeitos com TDA/H. Para isso, avaliou-se a atenção selectiva em sujeitos com e sem TDA/H, numa população de 183 sujeitos, em que 152 não apresentam e 31 apresentam TDA/H. Os resultados gerais comprovam que os sujeitos com TDA/H apresentam piores capacidades de atenção selectiva do que os sujeitos sem TDA/H, nomeadamente ao nível dos acertos, dos erros, da capacidade de concentração e do número de elementos processados.

Palavras-chave: Processos da atenção; Atenção selectiva; Transtorno do deficit de atenção com ou sem hiperactividade (TDA/H).

Abstract

The attention deficit is the major factor of Attention Deficit Hyperactivity Disorder (ADHD). This paper aims to demonstrate difficulties in selective attention of subjects with ADHD as compared to subjects without ADHD, attempting to verify which mechanisms of selective attention are more affected in subjects with ADHD. For this purpose, we evaluated selective attention in subjects with and without ADHD (152 without and 31 with ADHD), in a population of 183 subjects. The general results show that the subjects with ADHD present lower selective attention skills than the subjects without ADHD, specifically as far as rightness, errors, concentration and processed elements are concerned.

Keywords: Attention processes; Selective attention; Attention deficit hyperactivity disorder (ADHD).
\end{abstract}

Os seres humanos apresentam a capacidade de se adaptarem ao meio ambiente através das actividades mentais que desenvolvem, quer sejam de carácter perceptivo, representativo, da memória, ou outras, apresentando cada uma destas actividades uma função específica e concreta. A explicação para o facto dos seres humanos apresentarem as aptidões necessárias para realizarem tantas actividades conjuntas ou em simultâneo, reside nos processos da atenção, percebendo-se assim toda a sua importância.

Desde o nascimento que os aspectos sensoriais são a fonte de informação para desenvolver a atenção, sendo que o acto de atender consiste em focalizar selectivamente a consciência, filtrando e desejando informação não desejada, já que como não é possível atender a todos os estímulos de uma só vez, é necessário que exista um processo selectivo. Durante a atenção, os sujeitos acumulam a informação do meio externo, enquanto que a memória a curto prazo armazena e restaura essa mesma informação (Grippo \& Grippo, 2001).

\footnotetext{
* Endereço para correspondência: Universidade Fernando Pessoa, Praça 9 de Abril, 349, Porto, Portugal, 4249-004. E-mail: ramalho@ufp.pt
}

Embora existam várias terminologias que servem para designar os processos da atenção, nem todos os aspectos que estão directamente relacionados com este processo são sinónimos. Os actos de atender ou escutar, de observar ou perceber, não têm o mesmo significado, no entanto a comunidade científica tem vindo a reconhecer a importância não só da atenção como de todos os actos que com ela estão relacionados (García-Sevilla, 1997; Styles, 2006).

A atenção é um processo essencial no funcionamento cognitivo, quer seja para explorar o ambiente, de forma a manter focalizados os recursos perceptivos e cognitivos numa determinada estimulação, quer seja para seleccionar de entre os diversos estímulos recebidos quais os mais significativos para a situação actual. Está presente em qualquer tipo de actividade cognitiva seja voluntária ou não. A manifestação de dificuldades ao nível de sistemas atencionais, implicam diferenças substanciais ao nível da quantidade e da qualidade de informação que os sujeitos poderão processar.

Uma das primeiras referências às perturbações da atenção data de finais do séc. XIX, quando o investigador Ribot (1889) mencionou que são classificados de distraídos os sujeitos que são incapazes de se centrar de 
uma forma estável passando, incessantemente de uma ideia para outra.

Em 1902, o investigador Still, descreveu pela primeira vez, um grupo de 20 crianças com graus de agressividade, de hostilidade, de conduta desafiante, de desatenção e de agitação motora com o objectivo de conceptualizar uma síndrome que possa agrupar este conjunto de sintomas, de forma a obter uma explicação convincente da etiologia e sintomatologia deste transtorno (Capdevilla-Brophy et al., 2005; Herreros, Sánchez, Ajoy, \& García, 2002). Neste período, as aproximações científicas ao tema das perturbações da atenção e da actividade motora excessiva, essencialmente derivadas de investigações de carácter bioquímico, passam a ser designadas por impulsividade orgânica (Herreros et al., 2002) e por Disfunção Cerebral Mínima que designa uma perturbação no desenvolvimento do cérebro infantil, na qual as associações neuronais, responsáveis por determinadas funções, não maturam de acordo com a idade da criança (Veciana, 2002).

No fim dos anos 70 e início dos 80 surgem os manuais de diagnóstico e de classificação, como no DSMII (American Psychiatric Association [APA], 1968) e a CIE-9 (Organização Mundial de Saúde [OMS], 1978) com o intuito de tornar a denominação mais consensual, tomando a designação de "reacção hipercinética da infância", para o DSM-II (APA, 1968) e como "síndrome hipercinético da infância” para a CIE-9 (OMS, 1978).

No decorrer dos anos 80, surge, através do autor Barkley (1982), uma visão mais cognitivista das perturbações da atenção e da actividade motora excessiva, a qual procura funcionar como uma alternativa ao modelo comportamental. Desta forma, o diagnóstico das perturbações da atenção para a CIE-10 (OMS, 1992) fundamenta-se em critérios como a presença conjunta de sintomas básicos, como sejam a falta de atenção, inquietação e mobilidade excessiva, a estimativa da gravidade dos problemas, tendo como ponto de referência e idade e as capacidades intelectuais dos sujeitos, a avaliação do traço permanente ou situacional das alterações, a observação directa da sintomatologia, a ausência de psicose e distúrbio afectivo e o início precoce e persistência temporal da sintomatologia (Capdevilla-Brophy et al., 2005).

Actualmente, a definição e a configuração das perturbações da atenção estão envoltas em alguma discussão. A APA (2002), no DSM-IV-TR, considera que os principais sintomas das perturbações da atenção são o déficit de atenção, a hiperactividade e a impulsividade, enquanto que a CIE-10 ratifica unicamente os dois primeiros sintomas. Esta conjuntura contribui, naturalmente, para que exista uma certa ambiguidade e desordem no modo de realizar um diagnóstico apropriado.

O diagnóstico do TDA/H embora marcado, por vezes, por características difíceis de reconhecer, demonstra consenso científico quando se fala da sua prevalência, situando-a, na população escolar, entre os $3 \%$ e os $5 \%$
(Capilla-González et al., 2005; Cardo \& Servera-Barceló, 2005; Maynard, Tyler, \& Arnold, 1999; Veciana, 2002), sendo marcadamente uma perturbação associada ao estilo de vida das sociedades desenvolvidas actuais o que, origina assim, uma maior necessidade de estudo sobre ela.

Segundo a APA (2002) existem então 5 critérios que definem este transtorno, os quais referem que a característica essencial do TDA/H é um padrão persistente de falta de atenção e/ou impulsividade-hiperactividade, com uma intensidade que é mais frequente e grave que o observado habitualmente em outros sujeitos, que alguns dos sintomas da hiperactividade-impulsividade ou de falta de atenção podem ser a causa de problemas que tenderão a aparecer antes dos sete anos de idade e devem ocorrer em, pelo menos, dois contextos. Referem também que devem existir provas clarificadoras de um déficit clinicamente significativo do funcionamento social, académico ou laboral e que a perturbação não ocorre exclusivamente durante uma Perturbação Global do Desenvolvimento, Esquizofrenia ou qualquer outra Perturbação Psicótica e não é melhor explicada por outra Perturbação Mental. Os sintomas de presença do TDA/H devem persistir durante, pelo menos, 6 meses com uma intensidade que deverá ser desadaptativa e inconsistente, em relação com o nível de desenvolvimento do sujeito avaliado.

O TDA/H compreende três subtipos distintos, que são: (a) TDA/H, Tipo Misto onde a agitação psicomotora e o déficit de atenção estão presentes e ambos são significativos (Cardo \& Servera-Barceló, 2005; Sell-Salazar, 2003; Van-Wielink, 2005); (b) TDA/H, Tipo Hiperactivo-Impulsivo, onde são relevantes a hiperactividade e a impulsividade; (c) TDA/H, Tipo Desatento, no qual predomina a desatenção, em que estas crianças são referidas essencialmente como distraídas (Van-Wielink, 2005) e onde a hiperactividade não é significativa (Doyle, Faraone, DuPre, \& Biederman, 2001; Sell-Salazar, 2003).

Decorrente dos subtipos e dos critérios de diagnóstico referidos, este transtorno tem principais comorbilidades, entre outras, de cariz comportamental (desatenção, déficit de auto-controlo e inquietação), social (dificuldades de relacionamento interpessoal), cognitivo (dificuldades de selecção e manutenção da codificação de estímulos), académica (baixo rendimento escolar, problemas de comportamento), emocional (baixa auto-estima, baixa tolerância à frustração, alterações de humor) e físicas (alergias, alterações do sono, baixa coordenação motora) (Martínez, 2004).

Nos últimos anos, vários estudos não experimentais têm vindo a ser realizados de modo a verificar as competências atencionais em sujeitos diagnosticados com TDA/H. Os processos atencionais que têm vindo a ser os principais alvos desses estudos são os processos atencionais controlados ou conscientes, nomeadamente ao nível da atenção selectiva e também da atenção sustentada (García-Ogueta, 2001; Orjales, 1999; Ramalho, 2005). 
Decorrente das investigações realizadas, tem-se comprovado as dificuldades atencionais dos sujeitos com TDA/H. Ao nível da atenção selectiva (Gratch, 2000) estas dificuldades estão directamente relacionadas com o facto dos sujeitos com esta perturbação manifestarem uma baixa capacidade de tolerância à frustração e um elevado índice de impulsividade, que lhes origina dificuldades em, por exemplo, discriminar os estímulos significantes dos insignificantes. Segundo os autores M. Fernández e Deaño (2004), os sujeitos com TDA/H apresentam dificuldades atencionais que se reflectem na sua incapacidade para mudar, de uma forma adequada e deliberada, o foco atencional, uma vez que se apresentam como despistados, lentos, apáticos e descuidados.

Nas investigações realizadas por Baumeister (2003), o autor comprovou idênticas dificuldades atencionais no TDA/H. Verificou que os sujeitos com esta perturbação apresentam processos cognitivos e comportamentais de passividade, de processamento lento da informação, de confusão mental e por uma recuperação inconsciente da informação da memória, quando comparados com os sujeitos que não apresentam TDA/H.

Num outro estudo realizado, com o objectivo de comparar as capacidades atencionais em sujeitos com e sem TDA/H, particularmente ao nível do processo da atenção selectiva, os autores M. Pérez e Lago (2000) referem que as capacidades de reconhecimento, identificação e selecção da informação são bastante afectadas nos sujeitos com TDA/H, uma vez que estes apresentam mais dificuldades quando são colocados na tarefa estímulos insignificantes, resultantes das dificuldades que possuem em captar os detalhes, integrá-los e codificá-los adequadamente.

O desenvolvimento científico relacionado com a avaliação das capacidades de atenção no TDA/H e os resultados obtidos com estas investigações, abordam frequentemente a presença do déficit atencional nesta perturbação, no entanto, poucos são os estudos que procuram objectivar qual o processo da atenção específico e quais os mecanismos associados que se encontram afectados. Desta forma e, decorrente dos dados mencionados anteriormente, esta investigação tem como principais objectivos procurar verificar se existem diferenças significativas, na atenção selectiva, entre sujeitos com e sem TDA/H (Baumeister, 2003; M. Fernández \& Deaño, 2004; Gratch, 2000; M. Pérez \& Lago, 2000; Wilding, Munir, \& Cornish, 2001). Procura também veri-ficar, quais os mecanismos da atenção selectiva mais afectados nestes sujeitos, nomeadamente ao nível da qualidade de codificação de elementos significantes (acertos), insignificantes (erros), dos elementos significantes não assinalados (omissões) e da quantidade total de elementos processados. Esta análise, com características bem mais detalhadas, procura facilitar o processo de intervenção no TDA/H para este processo atencional.

\section{Método}

Nesta investigação foi utilizado um desenho de carácter não experimental, que tem como objecto de estudo verificar se a existência do TDA/H afecta os mecanismos gerais e específicos da atenção selectiva.

\section{Participantes}

A distribuição dos sujeitos da população em estudo situou-se entre o $3^{\circ}$ e o $9^{\circ}$ ano de escolaridade, num total de 183 , pertencentes a escolas públicas e privadas do distrito do Porto e de Aveiro, com idades compreendidas entre os 8 e os 16 anos.

A distribuição da amostra em função da idade, constitui-se em 31 (16,9\%) sujeitos com 8 anos, 22 (12\%) sujeitos com 9 anos, $21(11,5 \%)$ com 10 anos, $23(12,6 \%)$ com 11 anos, 30 (16,4\%) com 12 anos, 22 (12\%) com 13 anos, $23(12,6 \%)$ com 14 anos, $9(4,9 \%)$ com 15 anos e 2 $(1,1 \%)$ sujeitos com 16 anos.

Os sujeitos com TDA/H, embora estando diagnosticados previamente à investigação, foram alvo de uma nova avaliação através dos instrumentos Escala de Avaliação de Conners para Professores - Revista (Conners, 1997) e Escala de Avaliação do Déficit de Atenção com Hiperactividade (Farré \& Narbona, 2003), com intuito de comprovar o diagnóstico prévio existente. Ambos os instrumentos de diagnóstico serão abordados, em concreto, mais à frente. Desta forma, a distribuição da população em função do estatuto (com ou sem TDA/H), constituise em $31(16,9 \%)$ sujeitos com TDA/H e com $152(83,1 \%)$ sem TDA/H.

Em função do género, a população constitui-se em 84 sujeitos do sexo feminino $(45,9 \%)$ e 99 sujeitos do sexo masculino $(54,1 \%)$.

\section{Procedimento}

Antes da recolha de dados, foi pedido aos encarregados de educação a autorização de participação dos sujeitos na investigação, através da declaração de consentimento informado, na qual foi feita referência à confidencialidade e anonimato na aplicação das provas de avaliação.

As sessões de avaliação da atenção selectiva foram realizadas em grupo, com estes a serem constituídos em função de 2 parâmetros, as idades dos membros e a presença ou não do TDA/H. Estes parâmetros foram elaborados de modo a que cada um dos grupos fosse constituído por sujeitos com idades iguais ou aproximadas e também com o intuito de promover homogeneidade de cada grupo face à presença de TDA/H. Desta forma, foram constituídos 7 grupos de avaliação: um grupo com 31 sujeitos de 8 anos ( 5 com TDA/H), um grupo com 22 sujeitos de 9 anos (3 com TDA/H), um grupo com 21 sujeitos de 10 anos ( 4 com TDA/H), outro com 23 sujeitos de 11 anos ( 4 com TDA/H), um com 30 sujeitos de 12 anos (5 com TDA/H), outro com 22 sujeitos de 13 anos 
Ramalho, J. M. F., García-Señorán, M. M. \& González, S. G. (2009). Mecanismos de Atenção Selectiva na Perturbação de Hiperactividade com Déficit de Atenção.

(4 com TDA/H) e um grupo com 34 sujeitos de 14, 15 e 16 anos (6 com TDA/H).

As sessões de avaliação tiveram uma duração de 20 minutos aproximadamente. No final, os resultados foram comunicados aos sujeitos avaliados e aos encarregados de educação que solicitaram essa informação.

\section{Instrumentos}

A identificação de um sujeito com TDA/H requer um processo de diagnóstico complexo, onde deverá estar incluída toda a informação relevante, de modo a estabelecer uma correcta conceptualização da referida perturbação.

O diagnóstico dos sujeitos com TDA/H, foi realizado com a Escala de Avaliação de Conners para Professores - Revista (Conners, 1997) e com a Escala de Avaliação do Déficit de Atenção com Hiperactividade (Farré \& Narbona, 2003).

Escala de Avaliação de Conners para Professores (Conners, 1997). Abrange 3 sub-escalas, sendo elas, respectivamente (a) Comportamento Oposicional; (b) Problemas Cognitivos/Desatenção; (c) Hiperactividade; (d) $T D A / H$ Índex (que avalia Índice Geral de Hiperactividade). Nesta escala estão enumerados um conjunto de problemas comuns entre crianças em contexto escolar, organizada em 28 itens, avaliados através de uma escala de tipo Likert de 4 pontos, que reflectem a frequência com que o problema se tem manifestado no último mês, como sejam Nada Verdadeiro, Pouco Verdadeiro, Verdadeiro e Bastante Verdadeiro. A cotação é feita através do somatório de cada item. As respostas pontuadas em Nada Verdadeiro tem a cotação 0, por outro lado, tem a cotação máxima 3 quando assinalada a opção Bastante Verdadeiro e quanto maior for a pontuação que apresentarem mais frequentes serão os problemas que caracterizam as sub-escalas.

Escala de Avaliação do Déficit de Atenção com Hiperactividade (Farré \& Narbona, 2003). Permite uma medição dos sintomas do TDA/H e dos transtornos da conduta que podem co-existir com a referida perturbação. Tem aplicação individualizada, sendo a avaliação realizada através do professor. Tem uma duração variável, no entanto tem uma duração média entre 5 a $10 \mathrm{mi}$ nutos e tem como âmbito de aplicação sujeitos dos 6 aos 12 anos. É composta por 20 itens com duas sub-escalas de 10 itens cada: (a) Hiperactividade e Déficit de Atenção; (b) Transtornos da Conduta. A avaliação dos sujeitos consiste em colocar o aluno numa escala gradativa, em que o valor 0 corresponde a nada, o valor 1 corresponde a pouco, o valor 2 corresponde a bastante e o valor 3 corresponde a muito. Quanto maiores forem os valores, maior será o risco de TDA/H e Transtornos de Conduta.

$\mathrm{Na}$ avaliação da atenção selectiva foi utilizado o teste de atenção D2 (Brickenkamp, 1962), adaptado para versão espanhola por Seisdedos (2002). Esta prova tem uma aplicação que pode ser individual ou colectiva e tem como público de aplicação crianças, adolescentes e adultos. Tem como duração total de 8 a 10 minutos e apresenta como finalidade avaliar os vários aspectos da atenção selectiva e da concentração. Consiste no encontrar de figuras iguais ao modelo, em que existem 14 linhas de figuras e os sujeitos, em 20 segundos, para cada linha de figuras, terão de encontrar o número máximo de figuras iguais ao modelo. Apresenta como sub-escalas as seguintes: a) $T R$ (número total de elementos processados); (b) Erros (equívocos realizados); (c) TA (total de acertos); (d) VAR (pontuação de variância, dada pela diferença entre a maior e a menor produtividade nas linhas do teste); (e) Omissões (quantidade de elementos relevantes não processados); (f) $C O N$ (relacionada com a capacidade de concentração e deriva do número de elementos relevantes correctamente assinalados a subtrair pelo número de elementos relevantes não assinalados); (g) TOT (total da atenção selectiva).

\section{Resultados}

Os resultados, na generalidade demonstram diferenças entre o grupo de sujeitos com e sem TDA/H, particularmente ao nível das capacidades de focalização da atenção, da concentração e do processamento adequado de elementos significativos e não significativos.

Na Tabela 1 estão mencionados os resultados de uma análise de variância realizada para comparar os diversos parâmetros da atenção selectiva em sujeitos com TDA/H e sujeitos sem esta perturbação. Como se pode comprovar, na maioria dos parâmetros medidos surgem diferenças estatisticamente significativas entre os dois grupos, excepto na variável VAR e na variável omissões.

Os resultados demonstram que para a variável que avalia o total da atenção selectiva, os sujeitos com TDA/H apresentam um resultado significativamente inferior aos sujeitos sem TDA/H $(F=6.639, p<.05)$. Os sujeitos sem TDA/H apresentam resultados médios de 32.82 , enquanto que os sujeitos com TDA/H apresentam resultados de apenas 19.23.

Para as variáveis acertos e erros, os sujeitos com TDA/ $\mathrm{H}$ também apresentam piores resultados do que os sujeitos sem TDA/H $(F=7.218, p<.01 ; F=11.291, p<.01)$. Como se pode observar na tabela, na variável acertos, a média apresenta um valor de 34.06 para os sujeitos sem TDA/H e um valor de 19.97 para os sujeitos com TDA/ $\mathrm{H}$, enquanto que na variável erros, as médias são de 41.07, para os sujeitos sem TDA/H e de 22.39 para os com TDA/H

Para a variável CON, a qual avalia a capacidade de concentração e deriva do número de elementos relevantes correctamente assinalados a subtrair pelo número de elementos relevantes que não foram assinalados dentro do quadrado correspondente, os sujeitos sem TDA/H também obtiveram resultados significativamente melhores do que os sujeitos com TDA/H $(F=9.210, p<.01)$. Os 
Tabela 1

Análise de Variância para as Variáveis da Atenção Selectiva em Sujeitos com e sem TDA/H

\begin{tabular}{|c|c|c|c|c|c|c|}
\hline Variáveis & Estatuto & Média & $N$ & $D P$ & $F$ & Sig. \\
\hline \multirow[t]{3}{*}{ TOT } & Sem TDA/H & 32.82 & 152 & 27.14 & 6.639 & .011 \\
\hline & TDA/H & 19.23 & 31 & 24.88 & & \\
\hline & Total & 30.52 & 183 & 27.19 & & \\
\hline \multirow[t]{3}{*}{ TR } & Sem TDA/H & 34.41 & 152 & 27.85 & 5.344 & .022 \\
\hline & TDA/H & 21.87 & 31 & 25.67 & & \\
\hline & Total & 32.27 & 183 & 27.83 & & \\
\hline \multirow[t]{3}{*}{ VAR } & Sem TDA/H & 44.77 & 152 & 29.00 & .000 & .988 \\
\hline & TDA/H & 44.68 & 31 & 31.46 & & \\
\hline & Total & 44.75 & 183 & 29.34 & & \\
\hline \multirow[t]{3}{*}{ Acertos } & Sem TDA/H & 34.06 & 152 & 26.95 & 7.218 & .008 \\
\hline & TDA/H & 19.97 & 31 & 24.68 & & \\
\hline & Total & 31.65 & 183 & 27.04 & & \\
\hline \multirow[t]{3}{*}{$\mathrm{CON}$} & Sem TDA/H & 31.93 & 152 & 24.56 & 9.210 & .003 \\
\hline & TDA/H & 17.48 & 31 & 21.79 & & \\
\hline & Total & 29.45 & 183 & 24.67 & & \\
\hline \multirow[t]{3}{*}{ Omissões } & Sem TDA/H & 46.27 & 152 & 30.35 & 1.950 & .164 \\
\hline & TDA/H & 37.84 & 31 & 31.90 & & \\
\hline & Total & 44.83 & 183 & 30.69 & & \\
\hline \multirow[t]{3}{*}{ Erros } & Sem TDA/H & 41.07 & 152 & 29.12 & 11.291 & .001 \\
\hline & TDA/H & 22.39 & 31 & 22.94 & & \\
\hline & Total & 37.87 & 183 & 28.97 & & \\
\hline
\end{tabular}

sujeitos com TDA/H obtiveram um resultado médio de apenas 17.48, enquanto que os sujeitos sem TDA/H obtiveram uma média de 31.93 .

Para a variável $T R$, a qual avalia diz respeito ao número total de elementos processados durante a realização do teste, pode-se verificar que também aqui, os sujeitos sem TDA/H obtiveram melhores resultados do que os sujeitos com TDA/H $(F=5.344, p<.05)$. Os sujeitos com TDA/H apresentam uma média de 21.87 , enquanto que nos sujeitos sem TDA/H a média e de 34.41, o que demonstra as dificuldades em organizar as tarefas e as actividades e em realizar esforços mentais substanciais, resultantes do déficit de atenção presente nesta perturbação.

Para as variáveis VAR e Omissões não se encontraram diferenças estatisticamente significativas entre os sujeitos com e sem TDA/H.

\section{Discussão e Conclusões}

As competências atencionais, na sua generalidade, demonstram-se afectadas nos sujeitos com TDA/H, particularmente ao nível do processo de atenção selectiva, que pela sua especificidade e pelo tipo de tarefas que envolve, é naturalmente prejudicada nestes sujeitos (Baumeister, 2003; M. Fernández \& Deaño, 2004; Gratch, 2000; M. Pérez \& Lago, 2000; Wilding et al., 2001).
Tendo em conta este facto, neste estudo, tal como foi mencionado de uma forma prévia, procurou-se investigar se os sujeitos com TDA/H apresentam piores capacidades de atenção selectiva do que os sujeitos sem a perturbação, procurando também demonstrar, de uma forma concreta e objectiva, quais são as áreas ou elementos específicos deste processo da atenção, que podem estar mais afectados, de modo a facilitar o desenho de futuras intervenções.

Os resultados obtidos demonstram e corroboram os estudos que têm vindo a verificar que os sujeitos com TDA/H apresentam maiores dificuldades atencionais, ao nível da selecção da informação, em consequência dos padrões característicos desta perturbação. Tal como se verificou em outros estudos, os sujeitos com esta perturbação evidenciaram, nesta investigação, dificuldades de regulação atencional, que se traduziram em défices de análise e de descodificação dos elementos significantes e insignificantes e de focalização da atenção (Baumeister, 2003; Brown, 2003; García-Ogueta, 2001; Orjales, 1999; M. Pérez \& Lago, 2000; Ramalho, 2005; Wilding et al., 2001). Estes sujeitos revelaram também maiores dificuldades em planificar o processamento de um elevado número de elementos significativos em curtos espaços temporais, o que se explicita através do manifestar de um maior número de erros e de um menor número de acertos quando comparados com os sujeitos que não apresentam esta perturbação, evidenciados pelas diferenças 
significativas encontradas nos índices de avaliação estudados.

Por outro lado, embora os resultados indiquem que, nos índices relativos à diferença entre a maior e a menor produtividade ao longo do teste (VAR) e no valor de elementos significativos não assinalados (omissões), não foram encontradas diferenças significativas na comparação entre os sujeitos com e sem TDA/H, estes elementos da atenção deverão ser também tidos em conta como possíveis factores deficitários no TDA/H. O que os sujeitos com esta perturbação revelaram nesta investigação é que, por vezes, demonstram assinalar os estímulos-alvo de uma forma quase aleatória e, assim sendo, o número elementos assinalados foi expressivo, o que reduz a probabilidade de omissões mas não traduz uma efectiva descodificação da informação relevante e da irrelevante, dado que os sujeitos acabam por assinalar quer os elementos significativos quer os não significativos.

Os resultados demonstram também que os sujeitos com TDA/H revelam pouca capacidade de activação cognitiva e de planificação na realização das tarefas, facto este que se evidencia através da comparação dos índices da quantidade de elementos processados.

Decorrente das dificuldades de regulação atencional sentidas pelos sujeitos com TDA/H nesta investigação, é importante que, em termos futuros, sejam desenvolvidas competências cognitivas nestes sujeitos, que fomentem o desenvolvimento de estratégias de auto-regulação (A. Fernández, 2003; Sparks, Javorsky, \& Phillips, 2004), que possam consequentemente promover a focalização da atenção e também o processamento e codificação da informação, através do desenvolvimento das funções de análise e de síntese, para que as dificuldades sentidas na organização de actividades que envolvam a realização de esforços mentais substanciais sejam atenuadas. Estas estratégias podem passar pela elaboração de metas, por parte do sujeito, relacionadas com a sua forma de agir em determinadas circunstâncias, para que permaneça atento ao seu comportamento e podem passar também pelo treino em auto-instruções, que consistem no seguimento de instruções autodirigidas, as quais facilitam a verbalização, a reflexão e avaliação da sua actuação na realização das tarefas. Estas técnicas são reconhecidamente válidas para sujeitos com TDA/H (Calderón, 2001; Meichenbaum \& Goodman, 1971, citados por García, 2001; Miranda-Casas, Luz, \& Fernández, 2001; E. Pérez, 1998) já que desenvolvem estratégias de modelagem interna, como sejam a observação e controlo do comportamento e a internalização cognitiva.

Espera-se que este tipo de intervenção, que inclua também formas de potenciar e motivar para a aprendizagem, permita desenvolver competências de promoção escolar, social e mesmo emocional.

A investigação cientifica deverá, futuramente, investigar se as dificuldades de atenção que os sujeitos com TDA/H manifestaram na atenção selectiva, também se manifestam num outro processo atencional, em concreto na atenção sustentada, que se traduz na manutenção da atenção por longos períodos de tempo. A investigação neste âmbito poderá permitir um conhecimento mais revelador acerca de factores como sejam a persistência na realização de tarefas, a intensidade da atenção e o esforço de execução.

\section{Referências}

American Psychiatric Association. (1968). DSM-II: Diagnostic and statistical manual of mental disorders. Washington, DC: Author.

American Psychiatric Association. (2002). DSM-IV-TR4: Manual de diagnóstico e estatística das perturbações mentais (4. ed. rev.). Lisboa, Portugal: Climepsi.

Barkley, R. (1982). Guidelines for defining hyperactivity in children (attention deficit disorder with hyperactivity). In B. B. Lahey \& A. E. Kazdin (Eds), Advances in clínical child psychology (Vol. 5, pp. 137-180). New York: Plenum Press.

Baumeister, I. (2003). Hiperactivo, impulsivo, distraido: me conoces?. New York: Guilford Press.

Brickenkamp, R. (1962). Aufmerksamkkeits-Belastungs-Test (D2) (N. Cubero, Trans.). Madrid, España: TEA.

Brown, T. (2003). Trastornos por déficit de atención y comorbilidades en niños adolescentes y adultos. Barcelona, España: Masson.

Calderón, C. (2001). Resultados de un programa de tratamiento cognitivo-conductual para niños con trastorno por déficit de atención con hiperactividad. Anuario de Psicología, 32(4), 79-98.

Capdevilla-Brophy, C., Artigas-Pallarés, J., Ramírez-Mallafré, A., López-Rosendo, M., Real, J., \& Obiols-Llandrich, J. (2005). Fenotipo neuropsicológico del trastorno de déficit de atención/hiperactividad: ¿existen diferencias entre los subtipos? Neurología, 40(Supl. 1), S17-S23.

Capilla-González, A., Pazo, P., Campo, P., Maestú, F., Fernández, A., Fernández-González, S., et al. (2005). Nuevas aportaciones a la neurobiología del trastorno por déficit de atención con hiperactividad desde la magnetoencefalografia. Neurología, 40(Supl. 1), S43-S47.

Cardo, E., \& Servera-Barceló, M. (2005). Prevalencia del trastorno de déficit de atención e hiperactividad. Neurología, 40(Supl. 1), S11-S15.

Conners, K. (1997). Escala de Avaliação de Conners para Professores-Revista (S). New York: Multi-Health Systems.

Doyle, A., Faraone, S., DuPre, E., \& Biederman, J. (2001). Separating attention deficit hyperactivity disorder and learning disabilities in girls: A familial risk analysis. American Journal of Psychiatry, 158, 1666-1672.

Farré, A., \& Narbona, J. (2003). Evaluación del trastorno por déficit de atención con hiperactividad. Madrid, España: TEA.

Fernández, A. (2003). Problemas de lenguaje con estudiantes con déficit atencional. Valencia, España: Servei.

Fernández, M., \& Deaño, M. (2004). Déficit de atención con hiperactividad (TDA/H), procesos PASS y eficacia de un programa de intervención cognitiva. Educación, Desarrollo y Diversidad, 7(3), 51-92.

García, I. (2001). Hiperactividade. Prevenção, avaliação e tratamento na infância. Lisboa, Portugal: McGraw-Hill. 
García-Ogueta, M. (2001). Mecanismos atencionales y síndromes neuropsicológicos. Neurología, 32(5), 463-467.

García-Sevilla, J. (1997). Psicología de la atención. Madrid, España: Síntesis.

Gratch, L. (2000). El trastorno por déficit de atención: clínica, diagnóstico y tratamiento en la infancia, la adolescencia y la adultez. Buenos Aires, Argentina: Medica Panamericana.

Grippo, J., \& Grippo, T. (2001). Síndrome hiperatencional en niños. Neurología, 33(12), 1149-1151.

Herreros, O., Sánchez, F., Ajoy, M., \& García, R. (2002). Evaluación de niños y adolescentes con trastorno por déficit de atención con hiperactividad/trastornos hipercinéticos. Psiquiatría Infanto-Juvenil, 19(4), 199-214.

Orjales, I. (1999). Déficit de atención con hiperactividadmanual para padres y educadores. Madrid, España: CEPE.

Martínez, M. (2004). Dificultades ligadas a la hiperactividad y al deficit de atención. Necessidades educativas especiales. Madrid, España: McGraw-Hill.

Maynard, J., Tyler, J., \& Arnold, M. (1999, September). Coocurrence of attention deficit disorder and learning disability: An overview of research. Journal of Instructional Psychology.

Miranda-Casas, A., Luz, L., \& Fernández, S. (2001). Trastornos por déficit de atención con hiperactividad: una guía práctica. Málaga, España: Aljibe.

Organização Mundial de Saúde. (1978). CIE-9: International classification of diseases (9nd ed.). Genebra, Schweiz: Author.

Organização Mundial de Saúde. (1992). CIE-10: Trastornos mentales y del comportamiento. Descripciones clínicas $e$ pautas para diagnostico. Madrid, España: Meditor.

Pérez, E. (1998). Enfócate: entrenamiento en focalización de la atención. Bizkaia, España: Grupo Albor.

Pérez, M., \& Lago, A. (2000). Escalas Magallanes de Atención Visual. Bizkaia, España: Grupo Albor.

Ramalho, J. (2005). Procesos controlados de atención: estudio exploratorio en los subtipos del trastorno de hiperactividad con déficit de atención. Educación, Desarrollo y Diversidad, $8(2), 19-42$.

Ribot, T. (1889). La psychologie de l'attention. Paris: Alcan.

Sparks, R., Javorsky, J., \& Phillips, L. (2004). College students classified with ADHD and the foreign language requirement. Journal of Learning Disabilities, 37(2), 169-178.

Seisdedos, N. (2002). D2. Madrid, España: TEA.

Sell-Salazar, F. (2003). Síndrome de hiperactividad y déficit de atención. Neurología, 37(4), 353-358.

Styles, E. (2006). The psychology of attention. New York: Psychology Press.

Van-Wielink, G. (2005). Déficit de atención con hiperactividad: estrategias, habilidades, diagnóstico y tratamiento. México, DF: Trillas.

Veciana, J. (2002). Los trastornos de la atención y la hiperactividad: diagnóstico y tratamiento neurofuncional y causal. Barcelona, España: Lebón.

Wilding, J., Munir, F., \& Cornish, K. (2001). The nature of atencional differences between groups of children differentiated by teacher ratings of attention and hyperactivity. British Journal of Psychology, 92, 357-371. 